\title{
Selection of an optimal portfolio with stochastic volatility and discrete observations
}

\author{
N. V. Batalova ${ }^{1}$, V. Maroussov ${ }^{2}$ \& F. G. Viens ${ }^{3}$ \\ ${ }^{1}$ Bank of America Securities, London, U.K. \\ ${ }^{2}$ Department of Physics, Purdue University, West Lafayette, IN, USA \\ ${ }^{3}$ Department of Statistics, Purdue University, West Lafayette, IN, USA
}

\begin{abstract}
We give a numerical method to calculate the optimal self-financing portfolio of stock and risk-free asset to maximize the wealth's expected future utility, in the case of stochastic volatility and discrete observations: the portfolio stock allocation is only allowed to change discretely in time at fixed time intervals. We use a particle-filtering and Monte-Carlo-type algorithm, which we implement forward in time in the case of power utility.

Keywords: portfolio optimization, stochastic volatility, particle filtering, MonteCarlo method, expected utility, diffusion processes, numerical implementation.
\end{abstract}

\section{Introduction and summary}

For many markets, the Black-Scholes (BS) model's basic assumption, that a stock's volatility is constant, is far from being satisfied. Empirical evidence for this inadequacy is known to include the so-called volatility smile for implied volatilities, and other phenomena not visible within the BS model. Many natural extensions posit the volatility itself is random; when it is a stochastic process, this is the stochastic volatility (SV) model, which we use in this article. We propose a systematic way to optimize a portfolio of continuous-time SV stock and risk-free asset using a discrete-time strategy, thereby offering a way to minimize transaction costs.

Section 2 presents a short overview of the optimal portfolio selection problem, and its position in the literature, including some references on stochastic volatility, and a short list of ways which have been suggested to tackle the issue of nonconstant volatility. Section 3 describes the SV model, along with tools needed to 
understand our portfolio optimization problem, including (i) optimal stochastic volatility filtering under discrete observations, and its interacting particle method; (ii) the solution, developed in Viens [12], of the discrete observation optimization problem using a Bellman principle and stochastic dynamic programming. Because the algorithm in Viens [12] is too complex to be implemented, we show, in the special case of power utility in Section 4 how to reduce the complexity tremendously by using a simple mathematical argument, and a reasonable additional approximation. In section 5, results of our numerical implementation are presented, showing that our method typically outperforms the standard HamiltonJacobi-Bellman solution based on the BS model (the classical case of R. Merton, see Bjork [1, Ch. 14]). Conclusions are drawn in Section 6.

\section{Scientific context}

In the SV class of models, volatility depend on a latent unobserved stochastic process, which can be interpreted as a rate at which new economic information is absorbed by the market. See Ghysels et al. [10] for a survey of SV, Fouque et al. [6] for a detailed study of SV option pricing under fast mean reversion, and Cont et al. [11] for a number of recent advances by various authors. The main question in much of these works is that of SV estimation, a challenging statistical problem. The methodology we have chosen is a Bayesian one, specifically that of optimal non-linear stochastic filtering, where the discretely observed stock prices contain the useful information: see Section 3. Other SV Bayesian techniques which do not intersect ours have been proposed. In Frey and Runggaldier [7], an entirely different type of filtering is performed using the information contained in random observation times. Another different kind of "filtering" is the statistical estimation, pioneered by D.B. Nelson (see Fornari and Mele [8]), of coefficients in time series models of ARCH/GARCH type, which approximate SV models for highfrequency observations. The "method of moments" can be considered as a further distinct type of filtering: see Gallant et al. [9].

The portfolio optimization problem, in its basic form, is to maximize the expected future wealth (as measured using a concave utility function) of a portfolio of stock and risk-free account, using a self-financing dynamic strategy. In the SV literature, little attention has been given to this question. The few solutions rely on the assumption of high-frequency or continuous trading, e.g. Fouque et al. [6, Chapter 10]. Only Viens [12] addresses the question with discretetime observation and trading, but gives no hope for a practical implementation, because the algorithm is akin to a finite difference method for a PDE whose state space has a desperately high dimension. Our paper is the first to give a solid practical algorithm for portfolio utility optimization under continuous-time SV with dynamic discrete time updating; we are also among the first (see also Florescu and [5], and Desai et al. [4]) to show how optimal stochastic volatility filtering can be useful numerically. 


\section{Stochastic volatility, discrete observations, and optimization}

In our partial information setting there is no means of obtaining an arbitrarily accurate estimate of stochastic volatility. This section provides the estimator given by the stochastic volatility filter and its particle method. We then show how this "smart" (interacting) Monte-Carlo-type algorithm, combined with a further Monte-Carlo algorithm for one-step portfolio maximization, approximates the optimization problem.

\subsection{The model and the problem}

Under the market's probability measure $\mathbf{P}$, the stochastic volatility model for the stock price $X$ and the risk-free asset $B$ is

$$
d X_{t}=X_{t} \mu d t+X_{t} \sigma\left(Y_{t}\right) d W_{t}, \quad B_{t}=e^{r t}
$$

for all $t \geq 0$, where $W$ is a Brownian motion, eqn (1) is in the stochastic Itô sense (see Bjork [1]), $\mu$ is the constant mean rate of return, and $\sigma\left(Y_{t}\right)$ is a deterministic function of a stochastic process $Y_{t}$ that satisfies a diffusion equation driven by another Brownian motion $Z$ with $\operatorname{corr}(W, Z)=\rho$ where $0 \leq|\rho|<1$, i.e.

$$
d Y_{t}=\alpha\left(Y_{t}\right) d t+\gamma\left(Y_{t}\right) d Z_{t} .
$$

The choice of the function $\sigma$ is not fundamental: many choices will yield models which are difficult to distinguish empirically. One commonly assumes $\sigma(x)=\exp (x)$. The choice of the law of $Y$ is crucial. A popular choice, esp. in the case of highly traded assets and indexes (see Fouque et al. [6]), is a fastmean-reverting process such as the Ornstein-Uhlenbeck process with a large $\alpha$, i.e.

$$
d Y_{t}=\alpha\left(m-Y_{t}\right) d t+\sqrt{\alpha} d Z_{t} .
$$

For simplicity of notation, we assume now and throughout that our observation times are the non-negative integer $i=0,1, \ldots N$, with $N$ our time horizon. For a fixed scenario $\bar{x}=\left(x_{0}, \ldots, x_{N}\right)$, let $\mathcal{F}_{i}^{\bar{x}}$ be the event $\mathcal{F}_{i}^{\bar{x}}:=$ $\left\{X_{0}=x_{0}, \ldots, X_{i}=x_{i}\right\}$ (information from observations up to time $i$ ). We will often use the notation $\bar{x}_{i}:=\left(x_{0}, \ldots, x_{i}\right)$. The stochastic volatility filtering problem is to estimate the conditional probability distribution $p_{i}^{\bar{x}}(d y):=\mathbf{P}\left[Y_{i} \in d y \mid \mathcal{F}_{i}^{\bar{x}}\right]$. This is called the optimal stochastic filter because it minimizes its distance to the actual value of $Y$ in the sense of least squares, i.e. using $L^{2}$ norms under conditional expectation given the observations $\mathcal{F}_{i}^{\bar{x}}$.

A portfolio is defined by $(a, b)=\left(a_{i}, b_{i}\right)_{i=0}^{N}$, where $a_{i}$ represents the number of units of stock $X$ in our portfolio at time $i$, and $b_{i}$ the number of units of the riskfree asset (one unit is worth one dollar at time 0 ). This portfolio's wealth process $\mathcal{W}$, with constant stock holdings in each time interval to ensure that no transaction costs are incurred, is thus for every $s \in[i, i+1]: \mathcal{W}_{s}=\mathcal{W}_{s}^{a_{i}, b_{i}}=a_{i} X_{s}+b_{i} B_{s}$. 
Using the convenient substitution $b_{i}=\left(\mathcal{W}_{i}-x_{i} a_{i}\right) e^{-r i}$, the self-financing condition now simply reads

$$
\mathcal{W}_{s}=a_{i} X_{s}+\left(\mathcal{W}_{i}-X_{i} a_{i}\right) e^{r(s-i)}
$$

for all $i=0,1,2, \cdots, N-1$, and for all $s \in[i, i+1]$. Since in addition the initial wealth $\mathcal{W}_{0}=w_{0}$ is known, we see that the system is defined solely using the control variable $a_{i}$ and the state variable $x_{i}$ in each interval. Let $U$ be an increasing and concave function on $\mathbf{R}_{+}$, the utility function. In this paper we will use the popular so called HARA case $U(w)=w^{p} / p$ for some $p \in(0,1)$. The only condition we must impose on the set $A_{0}$ of admissible sequences $\left(a_{i}\right)_{i}$ is that $a_{i}$ depend solely on what information is available, i.e. $a_{i}=a_{i}\left(w_{0}, \bar{x}_{i}\right)$. Our task is to find a dynamic portfolio $a^{*}=\left(a_{i}^{*}\right)_{i=1}^{n}$ that attains the supremum in the P-expectation

$$
V\left(0, x_{0}, w_{0}\right)=\sup _{a \in A_{0}} \mathbf{E}\left[U\left(\mathcal{W}_{N}^{a, b}\right) \mid X_{0}=x_{0}, \mathcal{W}_{0}=w_{0}\right]
$$

\subsection{Filtering with stochastic volatility}

Viens [12] can be consulted for a recursion formula for the filter $p_{i}(d y)$. However, this formula cannot be evaluated explicitly. The interacting particle (or smart Monte Carlo, or MCMC) algorithm established in Del Moral et al. [3], yields a decent approximation (order $n^{-1 / 3}$ ) of $p_{i}^{\bar{x}}(d y)$ as the empirical distribution of a family of $n$ particles $\left(Y_{i}^{k}\right)_{k=1}^{n}$ :

$$
\hat{p}_{i}^{\bar{x}}(d y):=\frac{1}{n} \sum_{k=1}^{n} \delta_{Y_{i}^{k}}(d y) .
$$

We refer to Florescu and Viens [5] for a complete description of the algorithm, and to Del Moral et al. [3] for a proof of the convergence result. Summarizing, let us note that the particles evolve according to the iteration of a two-step (selection/mutation) process. In the mutation process, they evolve independently according to the Euler approximations of appropriate diffusions (dynamics given by eqns (1) and (2)), with number of time steps $m=n^{1 / 3}$ in each interval of length 1. The proof presented in Del Moral et al. [3] assumes that $\rho=0$, and shows the convergence in $L^{1}$ of $\hat{p}_{i}^{\bar{x}}(f)$ to $p_{i}^{\bar{x}}(f)$ for deterministic bounded test functions $f$. The extension to the case of $\rho \in(-1,1)$ is stated in Viens [12]. Our simulations indicate that our particle filter is good at stabilizing quickly, following the actual mean-reverting signal $Y$, and converges relatively fast to the optimal filter; in fact, our empirical convergence speed seems to be on the order of $m^{-3 / 2}=n^{-1 / 2}$, which is considerably faster than the result in Del Moral et al. [3], and is consistent with the comments in that reference indicating that ergodic signals should see faster convergence. 


\section{Numerically implementable approximation}

We embed our portfolio optimization problem (5) at time 0 into a dynamic one as follows: for all $w, x, \bar{x}$, for all $i=1,2, \ldots, N$, for all $s \in[i, i+1]$, find

$$
V(s, x, w)=V\left(s, x, w ; \bar{x}_{i}\right)=\sup _{a \in A_{0}} \mathbf{E}\left[U\left(\mathcal{W}_{N}^{a}\right) \mid X_{s}=x, \mathcal{W}_{s}^{a}=w, \mathcal{F}_{i}^{\bar{x}}\right] .
$$

A discrete Bellman principle was established in Viens [12] for the dynamic optimization problem (7), showing that the entire optimization can be solved by a discrete iteration of continuous HJB equations in the individual successive time intervals. Unfortunately, implementing this iteration backwards in time starting from $V\left(N, \bar{x}_{N}, w\right)=U(w)$ is essentially hopeless because it is akin to a finitedifference method whose state variable $\bar{x}_{i}$ has a time-dependent dimension which is extremely high even for moderate $i$.

In order to circumvent this problem, we take advantage of the HARA case $U(w)=w^{p} / p$ which features the possibility of preserving, approximately, the power form of $V$ in the parameter $w$. With such an approximation, we will see that a time-forward algorithm can be developed in order to calculate the optimal strategy $a_{i}^{*}\left(w, \bar{x}_{i}\right)$ directly, without needing to know $V\left(i, \bar{x}_{i}, w\right)$. If one then wishes to find the initial maximum expected future utility, one then only needs to use the optimal strategies $a^{*}$ computed for a number of scenarios, keeping track of the corresponding wealth processes, so that $V\left(0, w_{0}, x_{0}\right)$ can be obtained a posteriori by any Monte-Carlo method in a low-complexity way directly from eqn (5), replacing the supremum by the evaluation for $a^{*}$.

\subsection{Mathematical analysis}

The SV filtering algorithm results in a sequence indexed by time $i$ of a set of $n$ pairs of particles $\left(\hat{X}_{i}^{k}, Y_{i}^{k}\right)_{k=1}^{n}$ which approximate the distribution of $\left(X_{i}, p_{i}^{\bar{X}}\right)$ given the observations $\bar{x}_{i}$. Assume that we have constructed, as in Viens [12], an algorithm using these particles that outputs functions $\hat{V}\left(i, \bar{x}_{i}, w\right)$ as an approximation to $V\left(i, \bar{x}_{i}, w\right)$. In accordance with the Monte-Carlo method of [12], using eqn (4), and the notation

$$
\hat{\beta}_{k}\left(\bar{x}_{i}\right):=\hat{X}_{i+1}^{k}\left(\bar{x}_{i}\right)-x_{i} e^{r},
$$

one can check we must have

$$
\hat{V}\left(i, \bar{x}_{i}, w\right)=\max _{a \in \mathbf{R}} \frac{1}{n} \sum_{k=1}^{n} \hat{V}\left(i+1, \bar{x}_{i}, \hat{X}_{i+1}^{k}, a \hat{\beta}_{k}\left(\bar{x}_{i}\right)+w e^{r}\right) .
$$

We now prove that $\hat{V}\left(i+1, \bar{x}_{i+1}, w\right)=\left(w^{p} / p\right) K\left(i+1, \bar{x}_{i+1}\right)$ for some function $K$, using induction. For $i=N, \hat{V}\left(N, \bar{x}_{N}, w\right)=w^{p} / p$ obviously has the correct form with $K_{N} \equiv 1$. Plugging this form for $\hat{V}(i+1, \cdot)$ above, to evaluate 
$\hat{V}(i, \cdot)$ by evaluating the extremum, we only need to consider the zeros of the derivative with respect to $a$ for the resulting function, namely those $a=w e^{r} \lambda$ where $\lambda$ solves

$$
\sum_{k=1}^{n} K\left(i+1, \bar{x}_{i}, \hat{X}_{i+1}^{k}\right) \hat{\beta}_{k}\left(\bar{x}_{i}\right)\left(\lambda \hat{\beta}_{k}\left(\bar{x}_{i}\right)+1\right)^{p-1}=0 .
$$

The derivative w.r.t. $\lambda$ of the quantity above is always negative, which proves the sole extremum $\lambda=\lambda^{*}$ does correspond to a maximum $a^{*}=a_{i}^{*}\left(w, \bar{x}_{i}\right)=w e^{r} \lambda^{*}$ in eqn (9). This allows us to conclude

$$
\begin{aligned}
\hat{V}\left(i, \bar{x}_{i}, w\right) & =\frac{w^{p}}{p} e^{r p} \frac{1}{n} \sum_{k=1}^{n} K\left(i+1, \bar{x}_{i}, \hat{X}_{i+1}^{k}\right)\left(\lambda^{*} \hat{\beta}_{k}\left(\bar{x}_{i}\right)+1\right)^{p} \\
& =: \frac{w^{p}}{p} K\left(i, \bar{x}_{i}\right) .
\end{aligned}
$$

Since $\lambda^{*}$ solving eqn (10) depends only on $\bar{x}_{i}$ and the particles, not on $w$, the same holds for $K_{i}$ in the last expression above, which also serves as a backward induction formula to calculate $K_{i}$. Also note that since $w_{i}$ depends only on $w_{0}$ and $\bar{x}_{i}$, the same holds for $a^{*}$.

\subsection{Simplification: our algorithm}

In order to make the above solution forward in time, assume for the moment that the quantity $K\left(i+1, \bar{x}_{i+1}, \hat{X}_{i+1}^{k}\right)$ does not in fact depend on $k$. Then $\lambda^{*}$ is the unique solution of

$$
0=\sum_{k=1}^{n} \hat{\beta}_{k}\left(\bar{x}_{i}\right)\left(\lambda^{*} \hat{\beta}_{k}\left(\bar{x}_{i}\right)+1\right)^{p-1}
$$

computable forward in time with only the knowledge of $\hat{\beta}_{k}\left(\bar{x}_{i}\right)$ which can be calculated via their definition (8) at the same time as the filter. Equation (12) is the only equation the portfolio manager needs to solve to find the approximate optimal allocation $a^{*}=w e^{r} \lambda^{*}$.

This assumption on $\hat{K}$ corresponds to approximating each $\hat{X}_{i+1}^{k}$ by their empirical average $\check{X}_{i+1}=\frac{1}{n} \sum_{k=1}^{n} \hat{X}_{i+1}^{k}$. The variance of the error of each such approximation (which, by the propagation-of-chaos results in Del Moral [2] are known to be approximately IID for reasonably large $n$ ) is on the order of the length of the time interval $\Delta t$ used to simulate $\hat{X}_{i+1}^{k}$ starting from the observed $x_{i}$. In our presentation, $\Delta t=1$, but this is only for convenience. The accumulation of these errors in eqn (11) yields a variance of order $n^{-2} n \Delta t=\Delta t / n$, showing that unless $\Delta t$ is quite large, the error introduced by this new approximation will be of a smaller order than the particle filtering error $n^{-1 / 3}$. A proper mathematical justification of this approximation would take us beyond the scope of this article. We now give a detailed summary of our new forward-only algorithm. Since this algorithm runs using a single sequence $\bar{x}$ of observations, we have suppressed the notation $\bar{x}$ throughout. 


\subsubsection{Preliminary steps}

Decide on a number of particles $n$ such that the error $1 / \sqrt{n}$ is satisfactorily small. Let $m=n^{1 / 3}$, the number of Euler time steps per each time step $[i, i+1]$. Let $\hat{V}(N, w)=w^{p} / p$. Let $X_{0}^{k}=x_{0}, Y_{0}^{k}=y_{0}^{k}$, for all $k=1, \cdots, n$. A preliminary filter can be run prior to starting the optimization (time 0 ), in order to generate realistic initial particles $y_{0}^{k}$ : it is a way to implement a "break-in" period for the filter, one after which the filter's stability property will eliminate any misspecification of its initialization.

\subsubsection{On-line time loop}

For all $i=0$ to $N-1$, repeat

Particle Filtering: use the method of Del Moral et al. [3, Section 5] (see Florescu and Viens [5]) to calculate the approximate filter $\hat{p}_{i}^{\bar{x}}$ from $\hat{p}_{i-1}^{\bar{x}}$, as the empirical measure of the $n$ particles $\left\{Y_{i}^{k}\right\}_{k=1}^{n}$.

Euler/Monte-Carlo step for the optimization: For each $k=1, \cdots, n$, let $\hat{X}_{i+1}^{k}$ be the endpoint $\hat{X}_{i}^{k}(m)$ of the $m$-step Euler method to simulate $\left(X_{i+1}, Y_{i+1}\right)$ using the $(X, Y)$ dynamics starting at time $i$ from the starting point $\left(x_{i}, Y_{i}^{k}\right)$, where $Y_{i}^{k}$ is the $k$-th particle of the filter $\hat{p}_{i}^{\bar{x}}$. Specifically, with $\left(\chi_{m}^{i, k}\right)$ and $\left(\zeta_{m}^{i, k}\right)$ independent families of IID standard normals, for all $j=0, \cdots, m-1$

$$
\begin{aligned}
& \hat{X}_{i}^{k}(j+1)=\hat{X}_{i}^{k}(j)+\hat{X}_{i}^{k}(j) \mu m^{-1}+\hat{X}_{i}^{k}(j) \sigma\left(\hat{Y}_{i}^{k}(j)\right) m^{-1 / 2} \chi_{m}^{i, k}, \\
& \hat{Y}_{i}^{k}(j+1)=\hat{Y}_{i}^{k}(j)+\alpha\left(\hat{Y}_{i}^{k}(j)\right) m^{-1}+\gamma\left(\hat{Y}_{i}^{k}(j)\right) m^{-1 / 2} \zeta_{m}^{i, k} .
\end{aligned}
$$

Maximization step: Evaluate the quantities $\hat{\beta}_{k}:=\hat{X}_{i+1}^{k}-x_{i} e^{r}$ and find the solution $\lambda=\lambda_{i}^{*}$ of $\sum_{k=1}^{n} \hat{\beta}_{k}\left(\lambda \hat{\beta}_{k}+1\right)^{p-1}=0$, using any numerical procedure known for finding a root of nonlinear equation, such as simulated annealing.

Portfolio selection. The portfolio manager changes stock and risk-free account allocations in a self-financing way to obtain $a_{i}^{*}=w_{i} e^{r} \lambda_{i}^{*}$ where $w_{i}$ is the current wealth before the allocation change.

\subsubsection{Computation of initial maximal expected future utilities (optional)}

In order to present possible objectives for the client, for each fixed initial wealth of interest $w_{0}$, the portfolio manager may run repeated simulations of Step 1 for a large number of varying stock scenarios $\bar{x}$, and then calculate the average of all terminal wealth utilities $\left(w_{N}\right)^{p} / p$ to yield a good approximation for $V\left(0, x_{0}, w_{0}\right)$.

\section{Numerical results}

We have coded our algorithm, implementing both steps 1 and 2 above. Our main code outputs the sequence of optimal strategy values $a_{i}^{*}\left(w_{0}, \bar{x}_{i}\right)$ and its corresponding dynamic wealth sequence $w_{i}\left(w_{0}, \bar{x}_{i}\right)$ for any given sequence of observed stock prices $\bar{x}$. In order to obtain the maximum expected future utility, as described in step 2 above, we have implemented a further Monte-Carlo method by simulating, independently of step 1 , a large number of sequences of stock prices 


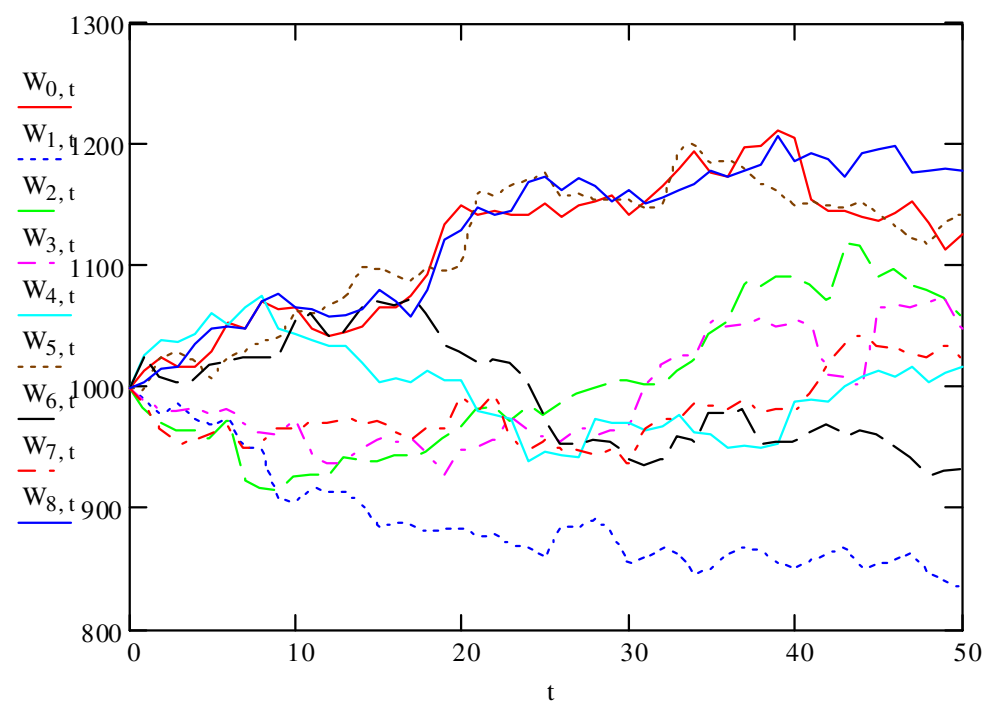

Figure 1: Optimal wealth $\mathcal{W}$ for eight typical scenarios. Params: $m=230$, $N=50, \sigma_{0}=0.25, \alpha=5, \mu=0.05, r=0.02, p=0.04$.

and volatilities following the true dynamics of $(X, Y)$ as in eqns (1), (2); each of these gives rise to a sequence $\bar{x}$ which is fed into the main code, resulting in a wealth at each time $i \leq N$. For $i=N$, these wealths are then injected into $U(w)$ and averaged yielding a Monte-Carlo approximation of $V\left(0, w_{0}\right)$. Using this approximation, we have been able to observe that a misspecification of the model, by which one assumes that volatility is constant (equal to the level of mean reversion), yields a significant decrease in expected utility, as one would hope. The wealth for a fixed scenario $\bar{x}$ can also be plotted individually (fig. 1), and compared to other strategies for the same scenario, such as pure stock, pure riskfree account, Merton's constant volatility scheme, and arbitrary randomly chosen strategies (see fig. 2). In fact, our fig. 2, which is typical of many scenarios for our choice of parameters, represents an average of dynamic utility over several scenarios. The solid red line corresponds to our method, while the dashed red line is Merton's method. We have strived to show pictures of typical situations, rather than repeating our algorithm until a favorable picture was obtained. This can be seen from the fact that the all-in-bank method (blue) outperforms many other strategies, because of poor stock performance. Nevertheless, our strategy is nearly systematically outperforming the constant-volatility method. It also does better than most random strategies. All-in-stock (brown) is clearly the loser in our simulated bearish market. As a measure of prudence, we have made sure that, when volatility is nearly constant (case of very small $\alpha$ ), our method yields, as it should, nearly the same portfolio as the Merton case (optimization for the BS model): $a^{*}=\sigma^{-2}(\mu-r) /(1-p)$, where $\sigma=\exp Y_{0}$ which we chose $\equiv 0.25$. 


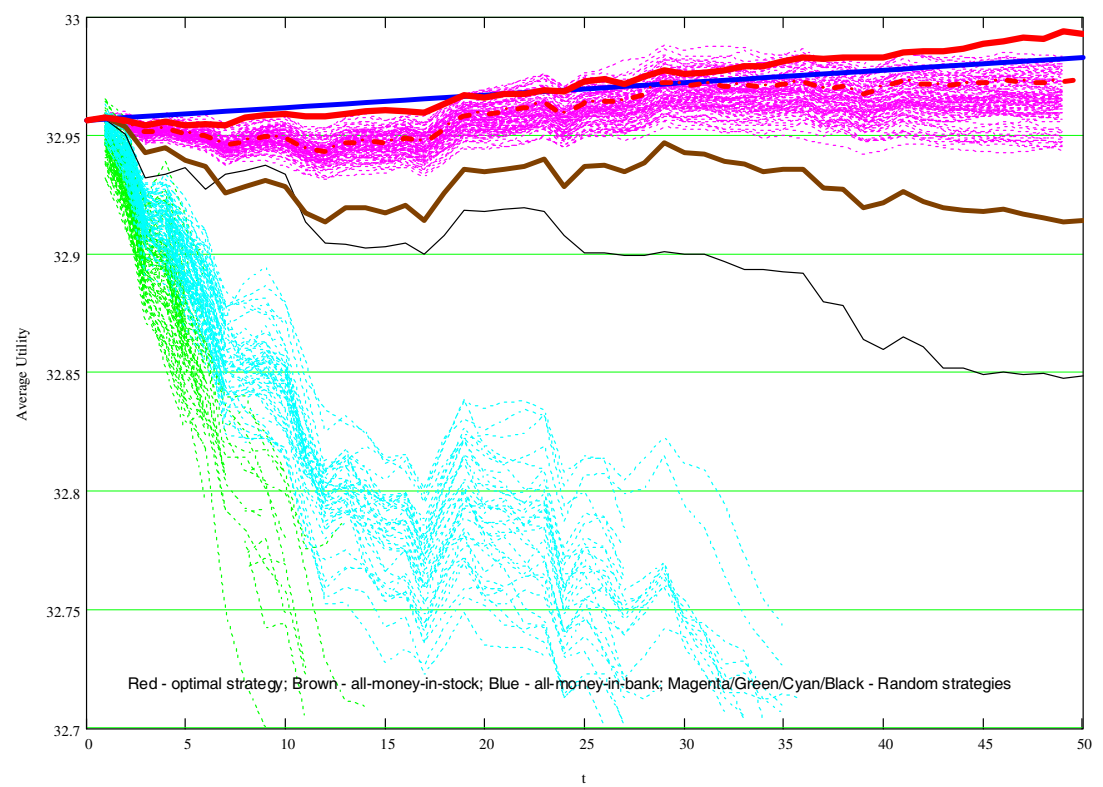

Figure 2: Dynamic average utility over 240 scenarios. Parameters are the same as in fig. 1.

\section{Conclusions}

Beyond solving the implementability issue for the algorithm of Viens [12] by way of an explicit calculation and an approximation tailored to the HARA case, our work has an important practical economic consequence. For investors who cannot observe and trade SV stock at high-frequency because of prohibitive transaction costs, rather than using an ad-hoc discrete adaptation of a continuously traded strategy, our algorithm provides the optimal portfolio selection method based solely, and dynamically, on moderate-frequency observations of a high-frequency asset price modeled by a continuous-time SV diffusion.

\section{References}

[1] Bjork, T. Arbitrage theory in continuous time. Oxford U.P., 1998.

[2] Del Moral, P. Feynman-Kac Formulae; Genealogical abd Interacting Particle Systems with Applications. Springer-V., 2004.

[3] Del Moral, P.; Jacod, J.; Protter Ph. The Monte Carlo method for filtering with discrete-time observations. Prob. Th. Rel. Fields. 120 (2001), no. 3, 346-368.

[4] Desai, R.; Lele, T.; Viens, F. A Monte-Carlo method for portfolio optimization under partially observed stochastic volatility. CIFEr 03 IEEE Int. Conf. on Comp. Intelligence for Fin. Eng. (2003), 257-263. 
[5] Florescu, I; Viens, F. Stochastic volatility: option pricing using a multinomial recombining tree. Preprint, 2006, available at http://www.stat.purdue.edu/ $/$ viens/quatrtree7.pdf.

[6] Fouque, J.-P.; Papanicolaou, G.; Sircar, K. R. Derivatives in financial markets with stochastic volatility. Cambridge University Press, Cambridge, 2000.

[7] Frey R.; Runggaldier W.G. A nonlinear filtering approach to volatility estimation with a view towards high frequency data. Int. J. Theor. Appl. Finance 4 (2001), no. 2, 199-210.

[8] Fornari, F.; Mele, A. Stochastic volatility in financial markets - Crossing the bridge to continuous time. Kluwer A.P., 2000.

[9] Gallant, A. R.; Hsieh, D.; Tauchen, G. E. Estimation of stochastic volatility models with diagnostics, Journal of Econometrics 81 159-192 (1997).

[10] Ghysels, E.; Harvey, A.C.; Renault, E. Stochastic volatility. Statistical methods in finance, 119-191, Handbook of Statist. 14, North-Holland, 1996.

[11] Special issue on volatility modelling. M. Avellaneda and R. Cont, eds. Quantitative Finance 2(1), 2002.

[12] Viens, F.G. Portfolio optimization under partially observed stochastic volatility. COMCON 8. W. Wells, Ed. 1-12. Optim. Soft., Inc, Pub. Div., 2002. 